\title{
ON TEMPERANCE AND RISKS SPREADING
}

\author{
Christophe Courbage, Geneva School of Business Administration (HES-SO) \\ Béatrice Rey, University Lyon, France
}

Abstract: This paper shows that temperance is the highest order risk preferences condition for which spreading $N$ independent and unfair risks provides the highest level of welfare than any other possible allocations of risks. These results are also interpreted through the concept of $N$-superadditivity of the utility premium. This paper provides a novel application of temperance, not in terms of two risks as it is common, but in terms of $N$ risks.

Keywords: temperance; risks spreading; superadditivity; utility premium

JEL classification: D81

\section{Introduction}

The concept of temperance, defined by the negative sign of the fourth derivative of the utility function, has been known for a little while in the literature. It was first introduced by Kimball (1992) to express a reduction of exposure to one risk in the presence of an unavoidable independent risk. Temperance is a useful notion as it has been shown to be a necessary condition for the important concept of risk vulnerability (Gollier and Pratt, 1996) as well as for the index of absolute prudence being decreasing in wealth (Gollier, 1996). More recently, Eeckhoudt and Schlesinger (2006) offered a context-free interpretation of temperance as a preference for disaggregation of two zero-mean risks. ${ }^{1}$ If a temperate individual faces two zero-mean independent risks, he prefers to face them separately rather than together. For instance, if a temperate decision-maker facing two zero-mean risks has to decide how to allocate these risks amongst two different areas or periods, he prefers to spread the two risks amongst the two areas or periods rather than to allocate them in one of the two. Hence temperance can express a preference for spreading of two zero-mean independent risks.

\footnotetext{
${ }^{1}$ Ebert et al. (2017) and Courbage et al. (2018) respectively reinterpret Eeckhoudt and Schlesinger (2006)'s results by showing that temperance ensures mutual aggravation, respectively mutual mitigation, of risk changes.
} 
The question we ask in this paper is what happens if the temperate decision-maker faces more than two risks and that these risks are not necessarily zero-mean risks. For instance, imagine that a temperate decision-maker faces three zero-mean independent risks and needs to decide whether he should face them the three together in the same area or period or faces each of them in a different area or period. Contrary to intuition, whatever the number of risks, preference for risks spreading does not require a higher order property than temperance. In particular, we show that conditions on the utility function for such preferences depend on the rules of dominance of the risks considered with respect to zero. We even show that not only spreading $N$ risks each over $N$ states rather than regrouping them in one state is welfare improving, but spreading risks each over $N$ states provides the highest level of welfare than any other possible allocations of risks between $N$ states. These results work even if risks are not indentically distributed.

The results of this paper can also be interpreted in terms of the property of superadditivity of the utility premium. To that aim, we define the concept of $N$-superadditivity of the utility premium and show that it is equivalent to have a decision-maker preferring to spread $N$ risks rather to regroup them in one state.

This paper therefore provides a new application of temperance, not in terms of two risks as it has been done in the litterature so far, but in terms of $N$ risks which is novel.

The paper is organised as follows. In section 2, we introduce the concept of temperance and its link with risk spreading. In section 3, we present our main results. In section 4, we reinterpret our results in terms of the property of superadditivity of the utility premium. Finally, the last section offers a short conclusion.

\section{Temperance and risks spreading}

Let consider an individual with initial wealth $x$ whose preferences are represented by a von Neumann-Morgenstern utility function $u$ that verifies no satiety $\left(u^{\prime}>0\right)$. This individual faces two independent zero-mean risks $X_{1}$ and $X_{2}$. Using the expected utility framework, Eeckhoudt and Schlesinger (2006) shows that a temperant individual (i.e. such that $\left.u^{\prime \prime \prime \prime}<0\right)$ prefers the equiprobable two states lottery i.e. the $50-50$ lottery $\left(X_{1}, X_{2}\right)$ to the $50-50$ lottery $\left(0, X_{1}+X_{2}\right)$ :

$$
\frac{1}{2} E\left[u\left(x+X_{1}\right)\right]+\frac{1}{2} E\left[u\left(x+X_{2}\right)\right] \geq \frac{1}{2} u(x)+\frac{1}{2} E\left[u\left(x+X_{1}+X_{2}\right)\right],
$$

or equivalently

$$
E\left[u\left(x+X_{1}\right)\right]+E\left[u\left(x+X_{2}\right)\right] \geq u(x)+E\left[u\left(x+X_{1}+X_{2}\right)\right] .
$$

A temperant individual prefers the left-hand side term of Eq. (2) to the right-hand side term because the pains are better apportioned. He prefers to allocate the risks in such a way as not to group the two risks in the same state.

Consider now the case where the individual faces three mutually independent zeromean risks, $X_{1}, X_{2}$ and $X_{3}$. What is the property of $u$ such that the individual prefers to spread the risks each over three states rather than regrouping them in a same state? More formally, what is the property of $u$ such that the individual prefers the equiprobable three states lottery $\left(X_{1}, X_{2}, X_{3}\right)$ to the equiprobable three states lottery $\left(0,0, X_{1}+X_{2}+X_{3}\right)$, 
i.e.

$\frac{1}{3} E\left[u\left(x+X_{1}\right)\right]+\frac{1}{3} E\left[u\left(x+X_{2}\right)\right]+\frac{1}{3} E\left[u\left(x+X_{3}\right)\right] \geq \frac{1}{3} u(x)+\frac{1}{3} u(x)+\frac{1}{3} E\left[u\left(x+X_{1}+X_{2}+X_{3}\right)\right]$,

or equivalently

$$
E\left[u\left(x+X_{1}\right)\right]+E\left[u\left(x+X_{2}\right)\right]+E\left[u\left(x+X_{3}\right)\right] \geq E\left[u\left(x+X_{1}+X_{2}+X_{3}\right)\right]+2 u(x) .
$$

Is temperance sufficient to obtain Eq.(4)? At first sight, the fact that $u^{\prime \prime \prime \prime}<0$ is sufficient is not straightforward. Indeed, on the one hand, risk aversion means that $E\left[u\left(x+X_{1}\right)\right] \leq$ $u(x), E\left[u\left(x+X_{2}\right)\right] \leq u(x)$ and then that $E\left[u\left(x+X_{1}\right)\right]+E\left[u\left(x+X_{2}\right)\right] \leq 2 u(x)$. On the other hand, since risks are mutually independent, risk aversion also means $E\left[u\left(x+X_{3}\right)\right] \geq$ $E\left[u\left(x+X_{1}+X_{2}+X_{3}\right)\right]$. By consequence, the term $E\left[u\left(x+X_{1}+X_{2}+X_{3}\right)\right]$ must be sufficiently smaller than the term $E\left[u\left(x+X_{3}\right)\right]$ to have Eq. (4) holding for all temperant decision-makers (DMs).

Note that Eeckhoudt and Schlesinger (2006) propose a way to apportion three mutually independent zero-mean risks considering equiprobable four states lotteries. They show that the equiprobable four states lottery $\left(0, X_{1}+X_{2}, X_{1}+X_{3}, X_{2}+X_{3}\right)$ is prefered to the equiprobable four states lottery $\left(X_{1}, X_{2}, X_{3}, X_{1}+X_{2}+X_{3}\right)$, i.e.:

$$
\begin{gathered}
\frac{1}{4} u(x)+\frac{1}{4} E\left[u\left(x+X_{1}+X_{2}\right)\right]+\frac{1}{4} E\left[u\left(x+X_{1}+X_{3}\right)\right]+\frac{1}{4} E\left[u\left(x+X_{2}+X_{3}\right)\right] \geq \\
\frac{1}{4} E\left[u\left(x+X_{1}\right]+\frac{1}{4} E\left[u\left(x+X_{2}\right)\right]+\frac{1}{4} E\left[u\left(x+X_{3}\right)\right]+\frac{1}{4} E\left[u\left(x+X_{1}+X_{2}+X_{3}\right)\right],\right.
\end{gathered}
$$

for a DM verifying $u^{(6)}<0$. Our framework is different as we consider equiprobable three states lotteries and not equiprobable four states lotteries to allocate the three risks. Furthermore, contrary to Eq. (5), we consider allocating each risk only once in one state.

So far, the case of spreading the three risks each over three states has been compared to the one of regrouping the three risks in the same state. Other allocations of risks between three states are possible in the sense that two risks can be regrouped in one state and the other risk in one of the two other states. Illustrations of such risks allocations are $\left(0, X_{1}+X_{2}, X_{3}\right),\left(0, X_{1}+X_{3}, X_{2}\right)$ and $\left(0, X_{2}+X_{3}, X_{1}\right)^{2}$. Therefore, a more general question is to know under which conditions spreading risks is preferred to any other possible allocations of risks between three states.

In this paper, we consider a DM facing $N$ mutually independent and unfair risks $X_{i}$ i.e. such that $E\left(X_{i}\right) \leq 0$ for all $i=1, \ldots, N$. We show that temperance is the highest order risk preferences condition for which risks spreading ${ }^{3}$ provides the highest level of welfare than any other possible allocations of risks.

A simple illustration of our framework is a DM who supervises $N$ similar geographical areas and who has to allocate the $N$ risks amongst the areas. This could be a government managing $N$ regions or a pharmaceutical firms launching a new drug in $N$ different countries. We could also imagine a clearing house allocating $N$ risks over $N$ different houses. Another illustration is a DM facing the $N$ risks in $N$ periods assuming a constant discount factor equals to one over time.

\footnotetext{
${ }^{2}$ Obviously, the lottery $\left(0, X_{1}+X_{2}, X_{3}\right)$ is equivalent in terms of welfare to the lottery $\left(X_{1}+X_{2}, 0, X_{3}\right)$ that is equivalent to the lottery $\left(X_{1}+X_{2}, X_{3}, 0\right)$ since lotteries are three state equiprobable lotteries.

${ }^{3}$ Risks spreading refers, throughout the paper, to spreading the $N$ risks each over $N$ states.
} 


\section{Main results}

Let's consider $N$ mutually independent and unfair risks $X_{i}\left(E\left(X_{i}\right) \leq 0\right.$ for all $i=$ $1, \ldots, N)$. We can distinguish two possible configurations of risks i.e. two possible statistical properties of risks $X_{i}$ : $(i)$ each risk $X_{i}$ is a pure risk i.e. a risk with all realisations strictly negative or equal to zero but never positive for which $E\left(X_{i}\right)<0 ;$ (ii) each risk $X_{i}$ is an unfair risk with realisations that can be positive, negative or equal to zero for which $E\left(X_{i}\right) \leq 0$. In each configuration, each risk can be characterised by a dominance property making it possible to compare random variables $X_{i}$ with 0 (i.e. no risk) via a statistical rule. In configuration $(i)$, each $X_{i}$ is dominated by 0 via first-order stochastic dominance $\left(X_{i} \preceq_{S D-1} 0 \forall i=1, \ldots, N\right)$. Obviously, in this case, $X_{i}$ can be a degenerated lottery such as $X_{i}=-k_{i}$ with $k_{i}>0 \forall i$. In configuration $(i i)$, as it is usual in economics since Rothschild and Stiglitz (1970), each $X_{i}$ is dominated by 0 via second-order stochastic dominance ${ }^{4}\left(X_{i} \preceq_{S D-2} 0 \forall i=1, \ldots, N\right)$. A particular case of $(i i)$ occurs when each $X_{i}$ is a zero-mean risk. In this case, each random variable $X_{i}$ is dominated by zero via second-order Ekern dominance $\left(X_{i} \preceq_{\text {Ekern-2 }} 0 \forall i=1, \ldots, N\right)$, which is a particular case of second-order stochastic dominance corresponding to the well-known mean-preserving increase in risk introduced by Rothschild and Stiglitz (1970).

For the sake of simplification, we adopt the notation $\left(\left\{X_{i}\right\}_{N}^{N}\right)$ to mean that the $N$ risks are allocated in the $N$ states of the equiprobable $N$ states lottery, i.e. $\left(X_{1}, X_{2}, \ldots, X_{N}\right)$. In terms of expected utility, this writes as $\frac{1}{N} \sum_{i=1}^{N} E\left[u\left(x+X_{i}\right)\right]$. The notation $\left(\left\{X_{i}\right\}_{N}^{N-1}\right)$ means that the $N$ risks are allocated in $N-1$ states of the equiprobable $N$ states lottery, $\left(\left\{X_{i}\right\}_{N}^{N-2}\right)$ means that the $N$ risks are allocated in $N-2$ states of the equiprobable $N$ states lottery, ..., $\left(\left\{X_{i}\right\}_{N}^{1}\right)$ means that the $N$ risks are allocated in one state.

Note that, except in the particular case where all risks $X_{i}$ are identically distributed, this notation does not allow to compare all risk allocations in terms of welfare. For instance, in the case where $N=3,\left(\left\{X_{i}\right\}_{3}^{2}\right)$ could mean $\left(0, X_{1}+X_{2}, X_{3}\right)$ or $\left(0, X_{1}+X_{3}, X_{2}\right)$ or $\left(0, X_{2}+X_{3}, X_{1}\right)$ i.e. in terms of welfare $u(x)+E\left[u\left(x+X_{1}+X_{2}\right)\right]+E\left[u\left(x+X_{3}\right)\right]$ or $u(x)+E\left[u\left(x+X_{1}+X_{3}\right)\right]+E\left[u\left(x+X_{2}\right)\right]$ or $u(x)+E\left[u\left(x+X_{2}+X_{3}\right)\right]+E\left[u\left(x+X_{1}\right)\right]$. Hence, in such a case, further assumptions on the statistical link between $X_{j}$ and $X_{k}$ for all $j, k=1,2,3$ and $j \neq k$ are required to compare the levels of welfare of these risk allocations.

To show that spreading risks provides the highest level of welfare that any other possible allocations of risks, we investigate the conditions on the utility function to obtain:

$$
\left(\left\{X_{i}\right\}_{N}^{N}\right) \succeq\left(\left\{X_{i}\right\}_{N}^{N-1}\right) \succeq \ldots \succeq\left(\left\{X_{i}\right\}_{N}^{2}\right) \succeq\left(\left\{X_{i}\right\}_{N}^{1}\right)
$$

Contrary to the intuition, whatever the number of risks $N(N \geq 2)$, conditions to obtain Eq. (6) do not require a higher order property than temperance. Indeed, we show that the statistical properties of $X_{i}$ govern the conditions on the utility function and do not involve a higher order property than a property of order 4 . We obtain the following

\footnotetext{
${ }^{4}$ Note that there exists cases where each risk $X_{i}$ is such that $E\left(X_{i}\right) \leq 0$ and where it is impossible to compare random variables $X_{i}$ with zero via a statistical rule. See Denuit et al. (1998) and Denuit et al. (2001) for more details.
} 
results (see proof in the Appendix).

Proposition 1. Given $N$ mutually independent and unfair risks $X_{i}\left(E\left(X_{i}\right) \leq 0\right.$ $\forall i=1, \ldots, N$ with $N \geq 2$ ), Eq. (6) holds under the following conditions:

(a) for all risk averse decision-makers $\left(u^{\prime}>0\right.$ and $\left.u^{\prime \prime}<0\right)$ when $X_{i} \preceq_{S D-1} 0$,

(b) for all mixed risk averse from 1 to 4 decision-makers $\left(u^{\prime}>0, u^{\prime \prime}<0, u^{\prime \prime \prime}>0\right.$ and $\left.u^{\prime \prime \prime \prime}<0\right)$ when $X_{i} \preceq_{S D-2} 0$.

Since zero-mean risks correspond to the particular case where $X_{i} \preceq_{E k e r n-2} 0$, we derive the following corollary from Proposition $1^{5}$.

Corollary 1. Given $N$ mutually independent and zero-mean risks $X_{i}\left(E\left(X_{i}\right)=0\right.$ $\forall i=1, \ldots, N$ with $N \geq 2)$, Eq. (6) holds for all temperant decision-makers $\left(u^{\prime \prime \prime \prime}<0\right)$.

In the case where $N=2$, note that our results coincide with the theorem of Eeckhoudt, Tsetlin and Schlesinger (2009). Note also that Proposition 1 holds whatever the signs of the derivatives of $u$ of order greater than 4 . Consequently, the results hold for all mixed-risk averse DMs $\left((-1)^{n+1} u^{(n)}>0 \forall n\right)$ i.e. for DMs who are risk-averse, prudent, temperant, edgy, etc. ${ }^{6}$

It should be stressed that Eq. (6) implies the following relation:

$$
\left(\left\{X_{i}\right\}_{N}^{N}\right) \succeq\left(\left\{X_{i}\right\}_{N}^{1}\right),
$$

that equivalently rewrites in terms of welfare as

$$
\sum_{i=1}^{N} E\left[u\left(x+X_{i}\right)\right] \geq(N-1) u(x)+E\left[u\left(x+\sum_{i=1}^{N} X_{i}\right)\right],
$$

meaning the DM prefers to spread the $N$ risks rather than to regroup them in one state. Surprisingly, this result is meaningful for management strategies of risk lover DMs. Indeed, in the case of zero-mean risks, Corollary 1 tells us that risk lover DMs can adopt the same risk management strategy as the one adopted by risk averse DMs, i.e. risk lover DMs can prefer to spread the $N$ risks rather than regroup them in one state and this, as soon as $N=2$. This happens as a risk lover DM can be temperant, for example, if his utility function writes as $u(x)=x^{\alpha}$ with $2<\alpha<3$ (see Ebert (2013) for more details).

\footnotetext{
${ }^{5}$ Note that under simple and usual regularity conditions $\left(u\right.$ defined over $\mathbb{R}^{+}$, non-satiation and bounded marginal utility tending to plus infinity), item (b) of Proposition 1 holds when the DM is only temperate without requiring risk aversion and prudence (see Menegatti (2014), Propositions 2 and 3). Indeed, under these simple conditions, Menegatti (2014) shows that prudence implies risk aversion and that temperance implies prudence.

${ }^{6}$ In item $(b)$ of Proposition 1, we assume that each $X_{i}$ is dominated by 0 via second-order stochastic dominance as it is usual in economics since Rothschild and Stiglitz (1970). Note that second-order stochastic dominance implies stochastic dominance of higher orders. Consequently, our results hold in cases where $X_{i} \preceq_{S D-s} 0 \forall s \geq 3$. If, instead of the Rothschild and Stiglitz assumption, we assume that $X_{i} \preceq_{S D-s} 0$ with $s \geq 3$, it is easy to show that item (b) of Proposition 1 writes then as follows: for all mixed risk averse from 1 to $2 s$ decision-makers $\left((-1)^{n+1} u^{(n)}>0 \forall n=1, \ldots, 2 s\right)$ when $X_{i} \preceq_{S D-s} 0$.
} 
Finally, let us make two important remarks. First, when we consider a number of risks higher than the number of states, e.g. $N$ risks and $M$ states with $N>M$, results of Proposition 1 still hold. Indeed, let's define, for example, $Y_{M}=X_{M}+\sum_{k=M+1}^{N} X_{k}$, and $Y_{i}=X_{i}$ for $i=1, \ldots, M-1$. From the property of $S D_{k}$ convolution stability (Denuit et al., 1998), $Y_{i} \preceq_{S D_{k}} 0$ for all $k=1,2$ and for all $i=1, \ldots, M$. The problem of the allocation of $N$ risks in $M$ states is then reduced to a problem of the allocation of $M$ risks $Y_{i}$ for all $i=1, \ldots, M$ in $M$ states. This reasoning holds whatever the distribution of the $N-M$ risks considered. Note that results of Proposition 1 also still hold when we consider a number of risks smaller than the number of states, e.g. $N$ risks and $M$ states with $N<M$. In that case, the best allocation is to allocate the $N$ risks in $N$ states, with $M-N$ states being allocated no risk.

The second remark is that Proposition 1 also holds when we consider the introduction, in each state ${ }^{7}$, of $L(L \geq 1)$ mutually independent background risks $\tilde{\epsilon}_{l}(l=1, \ldots L)$ which are assumed independent with $X_{i}$ for all $i$. To show this, we define the derived utility function (see Nachman (1982)) $v(x)=E\left[u\left(x+\sum_{l=1}^{L} \widetilde{\epsilon}_{l}\right)\right] \forall x$. The function $v$ shares the same properties as the utility function $u$ i.e. $\operatorname{Sign}\left\{v^{(n)}\right\}=\operatorname{Sign}\left\{u^{(n)}\right\}$ for all $n$ where $u^{(n)}$ denotes the derivative of order $n$ of the function $u$.

\section{$4 \quad N$-superadditivity of the utility premium}

In this section, we reinterpret our results in terms of the property of superadditivity of the utility premium. The utility premium is a tool introduced by Friedman and Savage (1948) initially to express a non-monetary measure of risk aversion.

When $N=2$, Eq. (6) writes as

$$
\left(\left\{X_{i}\right\}_{2}^{2}\right) \succeq\left(\left\{X_{i}\right\}_{2}^{1}\right)
$$

that equivalently rewrites as

$$
w\left(x ; X_{1}+X_{2}\right) \geq w\left(x ; X_{1}\right)+w\left(x ; X_{2}\right)
$$

where $w(x ; X)=u(x)-E[u(x+X)]$ denotes the utility premium defined by Friedman and Savage (1948). $w(x ; X)$ measures the degree of "pain" associated with facing the risk $X$, where pain is measured by the loss of welfare from adding the risk $X$ to wealth $x$. Indeed, for all risk $X$ such that $E(X) \leq 0, w(x ; X) \geq 0$ for all risk averse DMs. Eq. (10) means the supperadditivity of the utility premium, i.e. the pain of facing the two risks together is greater than the sum of the pain of facing each risk alone.

When the individual faces not two risks but $N$ risks, we can define the $N$-superadditivity of the utility premium as follows.

Definition. Given $N$ independent risks $X_{i}(i=1, \ldots, N$ with $N \geq 2)$, the utility premium is said $N$-superadditive when it verifies: $w\left(x ; \sum_{i=1}^{n} X_{i}\right) \geq \sum_{i=1}^{N} w\left(x ; X_{i}\right) \forall x$.

\footnotetext{
${ }^{7}$ In the case where the $L$ background risks are not introduced in each state, they can be then considered as additional risks. The DM faces then $N+L$ risks. If we assume $\tilde{\epsilon}_{l} \preceq_{S D_{k}} 0$ with $k=1,2$, i.e. if we assume that these risks share the same properties as $X_{i}$ for all $i$, this case brings us back to the previous remark (i.e. the case with a number of risks greater than the number of states).
} 
It is then easy to see that the $N$-superadditivity of the utility premium is equivalent to Eq. (8). We can then derive from Proposition 1 and Corollary 1 the following results.

Corollary 2. Given $N$ independent and unfair risks $X_{i}(i=1, \ldots, N$ with $N \geq 2)$, the utility premium is $N$-superadditive

(a) for all risk averse decision-makers $\left(u^{\prime}>0, u^{\prime \prime}<0\right)$ when $X_{i} \preceq_{S D-1} 0$,

(b) for all mixed risk averse from 1 to 4 decision-makers $\left(u^{\prime}>0, u^{\prime \prime}<0, u^{\prime \prime \prime}>0\right.$ and $u^{\prime \prime \prime \prime}<0$ ) when $X_{i} \preceq_{S D-2} 0$.

Corollary 3. Given $N$ independent zero-mean risks $X_{i}(i=1, \ldots, N$ with $N \geq 2)$, the utility premium is $N$-superadditive for all temperant decision makers $\left(u^{\prime \prime \prime \prime}<0\right)$.

\section{Conclusion}

This paper studies the conditions for which spreading $N$ independent and unfair risks provides the highest level of welfare than any other possible allocations of risks. Contrary to intuition, whatever the number of risks, preference for risks spreading does not require a higher order property than temperance, nor that risks are independent and identically distributed. In particular, we show that conditions on the utility function for such preferences depend on the rules of dominance of the risks considered with respect to zero.

The results of this paper can also be interpreted in terms of the property of superadditivity of the utility premium. To that aim, we define the concept of $N$-superadditivity of the utility premium and show that it is equivalent to have a decision-maker preferring to spread $N$ risks rather to regroup them in one state.

This paper provides a new interpretation of temperance and highlights its importance in individual decision making under risk. It also offers a novel application of temperance, not in terms of two risks as it has been done in the litterature so far, but in terms of $N$ risks.

These results find real-life applications in many ways. For instance, imagine a social planner who needs to allocate $N$ mutually independent and unfair risks, such as environmental risks, amongst a population composed of $N$ types of individuals representing each the same share of the population and each having the same wealth. This papers shows that if the social planner is temperant, then risks spreading is the best allocation. Extending our results to the cases where the share of the types of the population or the wealth of each type is not the same would be an interesting topic for future research. 


\section{Appendix - Proof of Proposition 1}

The proof uses the theorem of Eeckhoudt, Schlesinger and Tstelin (2009) and its corollary.

Theorem [Eeckhoudt, Schlesinger and Tsetlin (EST), 2009] Suppose that $Y_{i}$ dominates $\eta_{i}$ via $n_{i}$ th order stochastic dominance for $i=1,2\left(\eta_{1} \preceq_{S D_{n_{1}}} Y_{1}\right.$ and $\eta_{2} \preceq_{S D_{n_{2}}}$ $\left.Y_{2}\right)$ and suppose that $\eta_{1}, \eta_{2}, Y_{1}, Y_{2}$ are mutually independent risks. The $50-50$ lottery $\left[\eta_{1}+Y_{2}, Y_{1}+\eta_{2}\right]$ dominates the $50-50$ lottery $\left[\eta_{1}+\eta_{2}, Y_{1}+Y_{2}\right]$ via $\left(n_{1}+n_{2}\right)$ th-order stochastic dominance.

Corollary [Eeckhoudt, Schlesinger and Tsetlin (EST), 2009] Suppose that $Y_{i}$ dominates $\eta_{i}$ via $n_{i}$ th order Ekern dominance for $i=1,2\left(\eta_{1} \preceq_{\text {Ekern }_{n_{1}}} Y_{1}\right.$ and $\eta_{2} \preceq_{\text {Ekern }}$ $_{n_{2}}$ $\left.Y_{2}\right)$ and suppose that $\eta_{1}, \eta_{2}, Y_{1}, Y_{2}$ are mutually independent risks. The $50-50$ lottery $\left[\eta_{1}+Y_{2}, Y_{1}+\eta_{2}\right]$ dominates the $50-50$ lottery $\left[\eta_{1}+\eta_{2}, Y_{1}+Y_{2}\right]$ via $\left(n_{1}+n_{2}\right)$ th-order Ekern dominance.

When $Y_{1}=Y_{2}=0$ and using the Expected Utility Theory model, the EST (2009) theorem and its corollary respectively rewrite as follows:

EST (2009) Theorem: the inequality $E\left[u\left(x+\eta_{1}\right)\right]+E\left[u\left(x+\eta_{2}\right)\right] \geq u(x)+E\left[u\left(x+\eta_{1}+\eta_{2}\right)\right]$ holds for all utility function $u$ such that $(-1)^{(s+1)} u^{(s)}>0 \forall s=1, \ldots, n_{1}+n_{2}$.

EST (2009) Corollary: the inequality $E\left[u\left(x+\eta_{1}\right)\right]+E\left[u\left(x+\eta_{2}\right)\right] \geq u(x)+E\left[u\left(x+\eta_{1}+\eta_{2}\right)\right]$ holds for all utility function $u$ such that $(-1)^{\left(n_{1}+n_{2}+1\right)} u^{\left(n_{1}+n_{2}\right)}>0$.

In the case $N=2$, Proposition 1 and Corallary 1 respectively coincide with the EST theorem and its corollary. To prove the result in the case of $N$ risks with $N \geq 3$, we use the property of convolution stability of $S D_{k}$ (see Proposition 3-11 in Denuit et al. (1998) for more details):

Convolution stability property Suppose two random independent variables $X_{1}$ and $X_{2}$ such that $X_{1} \preceq_{S D_{k}} 0$ and $X_{2} \preceq_{S D_{k}} 0$ for all $k=1,2$, then $X_{1}+X_{2} \preceq_{S D_{k}} 0$ for all $k=1,2$.

We make the proof of Eq. (6) by steps. Let's rewrite Eq. (6) as follows:

$$
\left(\left\{X_{i}\right\}_{N}^{N}\right) \succeq_{1}\left(\left\{X_{i}\right\}_{N}^{N-1}\right) \succeq_{2}\left(\left\{X_{i}\right\}_{N}^{N-2}\right) \succeq_{3}\left(\left\{X_{i}\right\}_{N}^{N-3}\right) \succeq_{4} \ldots \succeq_{N-2}\left(\left\{X_{i}\right\}_{N}^{2}\right) \succeq_{N-1}\left(\left\{X_{i}\right\}_{N}^{1}\right)
$$

where indexes $1,2, \ldots, N-1$ indicate the different steps. In step 1 we show the first part of the preference relation, $\left(\left\{X_{i}\right\}_{N}^{N}\right) \succeq_{1}\left(\left\{X_{i}\right\}_{N}^{N-1}\right)$, in step 2 we show the second part of the preference relation, $\left(\left\{X_{i}\right\}_{N}^{N-1}\right) \succeq_{2}\left(\left\{X_{i}\right\}_{N}^{N-2}\right)$, in step 3 we show the third part of the preference relation, $\left(\left\{X_{i}\right\}_{N}^{N-2}\right) \succeq_{2}\left(\left\{X_{i}\right\}_{N}^{N-3}\right)$ etc.

$$
\underline{\text { Step } 1}:\left(\left\{X_{i}\right\}_{N}^{N}\right) \succeq_{1}\left(\left\{X_{i}\right\}_{N}^{N-1}\right)(P 1)
$$

The left-hand side term of $(P 1)$ writes as $\sum_{i=1}^{N} E\left[u\left(x+X_{i}\right)\right]$. In the set of $N$ risks $X_{i}$, we choose two risks that we name $X_{t}$ and $X_{l}$, and that we regroup into a single risk $X_{t}+X_{l}$ 
in the right-hand side term of $(P 1)$. Using this, $(P 1)$ rewrites as

$$
\sum_{i=1}^{N} E\left[u\left(x+X_{i}\right)\right]+E\left[u\left(x+X_{t}\right)\right]+E\left[u\left(x+X_{l}\right)\right] \geq \sum_{i=1}^{N} E\left[u\left(x+X_{i}\right)\right]+E\left[u\left(x+X_{t}+X_{l}\right)\right]+u(x)
$$

for all $t \leq N, l \leq N, t \neq l, i \neq t$ and $i \neq l$, that is equivalent to

$$
E\left[u\left(x+X_{t}\right)\right]+E\left[u\left(x+X_{l}\right)\right] \geq+E\left[u\left(x+X_{t}+X_{l}\right)\right]+u(x)
$$

which holds following $\operatorname{EST}(2009)$ theorem for all $u$ such that $(-1)^{(s+1)} u^{(s)}>0 \forall s=$ $1, \ldots, 2 k$ if $X_{1} \preceq_{S D_{k}} Y_{1}$ and $X_{2} \preceq_{S D_{k}} Y_{2}, k=1,2$. This inequality also holds following EST (2009) corollary for all $u$ such that $u^{(4)}<0$ in the particular case where risks are zero mean risks i.e. $X_{1} \preceq_{\text {Ekern }} Y_{1}$ and $X_{2} \preceq_{\text {Ekern }_{2}} Y_{2}$.

In the expression $\sum_{i=1}^{N} E\left[u\left(x+X_{i}\right)\right]+E\left[u\left(x+X_{t}+X_{l}\right)\right]+u(x)$ (with $t \leq N, l \leq N, t \neq l$, $i \neq t$ and $i \neq l)$, we can consider that we have $(N-1)$ risks. Indeed, let's write $X_{i}^{1}=X_{i}$ $\forall i=1, \ldots, N-2, i \neq t$ and $i \neq l$, and $X_{N-1}^{1}=X_{t}+X_{l}$, then the above expression can be equivalently rewriten as $\sum_{i=1}^{N-1} E\left[u\left(x+X_{i}^{1}\right)\right]+u(x)$ with $X_{i}^{1} \preceq_{S D_{k}} 0 \forall i$, for all $k=1,2$ following the convolution stability property. Using the same technique as the one used at the beginning of step 1 to rewrite $\sum_{i=1}^{N} E\left[u\left(x+X_{i}\right)\right]$, the expression $\sum_{i=1}^{N-1} E\left[u\left(x+X_{i}^{1}\right)\right]+u(x)$ can thus be equivalently rewriten as $\sum_{i=1}^{N-1} E\left[u\left(x+X_{i}^{1}\right)\right]+E\left[u\left(x+X_{t}^{1}\right)\right]+E\left[u\left(x+X_{l}^{1}\right)\right]+u(x)$ for all $t \leq N-1, l \leq N-1, i \neq t$ and $i \neq l$.

$\underline{\text { Step 2 }}:\left(\left\{X_{i}\right\}_{N}^{N-1}\right) \succeq_{2}\left(\left\{X_{i}\right\}_{N}^{N-2}\right)$

Following the same methodology as the one used in step $1,(P 2)$ rewrites equivalently as $\sum_{i=1}^{N-1} E\left[u\left(x+X_{i}^{1}\right)\right]+E\left[u\left(x+X_{t}^{1}\right)\right]+E\left[u\left(x+X_{l}^{1}\right)\right]+u(x) \geq \sum_{i=1}^{N-1} E\left[u\left(x+X_{i}^{1}\right)\right]+E\left[u\left(x+X_{t}^{1}+X_{l}^{1}\right)\right]+2 u(x)$

for all $t \leq N-1, l \leq N-1, t \neq l, i \neq t$ and $i \neq l$, that is equivalent to

$$
E\left[u\left(x+X_{t}^{1}\right)+E\left[u\left(x+X_{l}^{1}\right) \geq E\left[u\left(x+X_{t}^{1}+X_{l}^{1}\right)+u(x)\right.\right.\right.
$$

which holds following $\operatorname{EST}(2009)$ theorem for all $u$ such that $(-1)^{(s+1)} u^{(s)}>0 \forall s=$ $1, \ldots, 2 k$ if $X_{1} \preceq_{S D_{k}} Y_{1}$ and $X_{2} \preceq_{S D_{k}} Y_{2}, k=1,2$. This inequality also holds following EST (2009) corollary for all $u$ such that $u^{(4)}<0$ in the particular case where risks are zero mean risks i.e. $X_{1} \preceq_{E k e r n_{2}} Y_{1}$ and $X_{2} \preceq_{E k e r n_{2}} Y_{2}$.

In the expression $\sum_{i=1}^{N-1} E\left[u\left(x+X_{i}^{1}\right)\right]+E\left[u\left(x+X_{t}^{1}+X_{l}^{1}\right)\right]+2 u(x)$, we can consider that we have $(N-2)$ risks $X_{i}^{2}$ (i.e. $(N-3)$ risks $X_{i}^{1}$ and 1 risk $\left.X_{t}^{1}+X_{l}^{1}\right)$. Thus it can be equivalently rewriten as $\sum_{i=1}^{N-2} E\left[u\left(x+X_{i}^{2}\right)\right]+2 u(x)$ with $X_{i}^{2} \preceq S D_{k} 0$ for all $k=1,2$ following the convolution stability property. It can also be rewriten as $\sum_{i=1}^{N-2} E\left[u\left(x+X_{i}^{2}\right)\right]+E\left[u\left(x+X_{t}^{2}\right)\right]+E\left[u\left(x+X_{l}^{2}\right)\right]+2 u(x)$ for all $t \leq N-2, l \leq N-2$, $t \neq l, i \neq t$ and $i \neq l$.

$$
\text { Step 3 : }\left(\left\{X_{i}\right\}_{N}^{N-2}\right) \succeq_{3}\left(\left\{X_{i}\right\}_{N}^{N-3}\right)
$$

Following the same methodology as the one used in step 2, $(P 3)$ rewrites equivalently as $\sum_{i=1}^{N-2} E\left[u\left(x+X_{i}^{2}\right)\right]+E\left[u\left(x+X_{t}^{2}\right)\right]+E\left[u\left(x+X_{l}^{2}\right)\right]+2 u(x) \geq \sum_{i=1}^{N-2} E\left[u\left(x+X_{i}^{2}\right)\right]+E\left[u\left(x+X_{t}^{2}+X_{l}^{2}\right)\right]+3 u(x)$ 
for all $t \leq N-2, l \leq N-2, t \neq l, i \neq t$ and $i \neq l$, that is equivalent to

$$
E\left[u\left(x+X_{t}^{2}\right)+E\left[u\left(x+X_{l}^{2}\right) \geq+E\left[u\left(x+X_{t}^{2}+X_{l}^{2}\right)\right]+u(x)\right.\right.
$$

which holds following EST (2009) theorem for all $u$ such that $(-1)^{(s+1)} u^{(s)}>0 \forall s=$ $1, \ldots, 2 k$ if $X_{1} \preceq_{S D_{k}} Y_{1}$ and $X_{2} \preceq_{S D_{k}} Y_{2}, k=1,2$. This inequality also holds following EST (2009) corollary for all $u$ such that $u^{(4)}<0$ in the particular case where risks are zero mean risks i.e. $X_{1} \preceq_{\text {Ekern }_{2}} Y_{1}$ and $X_{2} \preceq_{\text {Ekern }} Y_{2}$.

In the expression $\sum_{i=1}^{N-2} E\left[u\left(x+X_{i}^{2}\right)\right]+E\left[u\left(x+X_{t}^{2}+X_{l}^{2}\right)\right]+3 u(x)$, we can consider that we have $(N-4)$ risks $X_{i}^{3}$ (i.e. $(N-2)$ risks $X_{i}^{2}$ and 1 risk $\left.X_{t}^{2}+X_{l}^{2}\right)$. Thus it can be equivalently rewriten as $\sum_{i=1}^{N-3} E\left[u\left(x+X_{i}^{3}\right)\right]+3 u(x)$ with $X_{i}^{3} \preceq_{S D_{k}} 0$ for all $k=1,2$ following the convolution stability property. It can also be rewriten as $\sum_{i=1}^{N-3} E\left[u\left(x+X_{i}^{3}\right)\right]+E\left[u\left(x+X_{t}^{3}\right)\right]+E\left[u\left(x+X_{l}^{3}\right)\right]+3 u(x)$ for all $t \leq N-3, l \leq N-3$, $t \neq l, i \neq t$ and $i \neq l$.

$\underline{\text { Step } m:}\left(\left\{X_{i}\right\}_{N}^{N-(m-1)}\right) \succeq_{m}\left(\left\{X_{i}\right\}_{N}^{N-m}\right)(P m)$

$(P m)$ rewrites equivalently as

$\sum_{i=1}^{N-(m-1)} E\left[u\left(x+X_{i}^{m-1}\right)\right]+E\left[u\left(x+X_{t}^{m-1}\right)\right]+E\left[u\left(x+X_{l}^{m-1}\right)\right]+(m-1) u(x) \geq$

$\sum_{i=1}^{N-(m-1)} E\left[u\left(x+X_{i}^{m-1}\right)\right]+E\left[u\left(x+X_{t}^{m-1}+X_{l}^{m-1}\right)\right]+m u(x)$

for all $t \leq N-(m-1), l \leq N-(m-1), t \neq l, i \neq t$ and $i \neq l$, that is equivalent to

$$
E\left[u\left(x+X_{t}^{m-1}\right)+E\left[u\left(x+X_{l}^{m-1}\right) \geq+E\left[u\left(x+X_{t}^{m-1}+X_{l}^{m-1}\right)\right]+u(x)\right.\right.
$$

which holds following EST (2009) theorem for all $u$ such that $(-1)^{(s+1)} u^{(s)}>0 \forall s=$ $1, \ldots, 2 k$ if $X_{1} \preceq_{S D_{k}} Y_{1}$ and $X_{2} \preceq_{S D_{k}} Y_{2}, k=1,2$. This inequality also holds following EST (2009) corollary for all $u$ such that $u^{(4)}<0$ in the particular case where risks are zero mean risks i.e. $X_{1} \preceq_{\text {Ekern }_{2}} Y_{1}$ and $X_{2} \preceq_{\text {Ekern }_{2}} Y_{2}$.

In the expression $\sum_{i=1}^{N-(m-1)} E\left[u\left(x+X_{i}^{m-1}\right)\right]+E\left[u\left(x+X_{t}^{m-1}+X_{l}^{m-1}\right)\right]+m u(x)$, we can consider that we have $(N-m)$ risks $X_{i}^{m}$ (i.e. $\left.(N-m-1)\right)$ risks $X_{i}^{m-1}$ and 1 risk $\left.X_{t}^{m-1}+X_{l}^{m-1}\right)$. Thus it can be equivalently rewriten as $\sum_{i=1}^{N-m} E\left[u\left(x+X_{i}^{m}\right)\right]+E[u(x+$ $\left.\left.X_{t}^{m}\right)\right]+E\left[u\left(x+X_{l}^{m}\right)\right]+m u(x)$ with $X_{i}^{m} \preceq_{S D_{k}} 0$ for all $k=1,2$ following the convolution stability property.

$$
\begin{aligned}
& \text { Step } N-2:\left(\left\{X_{i}\right\}_{N}^{3}\right) \succeq_{N-2}\left(\left\{X_{i}\right\}_{N}^{2}\right)(P(N-2)) \\
& (P(N-2)) \text { rewrites equivalently as } \\
& \sum_{i=1}^{N-(N-3)} E\left[u\left(x+X_{i}^{N-3}\right)\right]+E\left[u\left(x+X_{t}^{N-3}\right)\right]+E\left[u\left(x+X_{l}^{N-3}\right)\right]+(N-3) u(x) \geq \\
& \sum_{i=1}^{N-(N-3)} E\left[u\left(x+X_{i}^{N-3}\right)\right]+E\left[u\left(x+X_{t}^{N-3}+X_{l}^{N-3}\right)\right]+(N-2) u(x)
\end{aligned}
$$

for all $t \leq N-(N-3), l \leq N-(N-3), t \neq l, i \neq t$ and $i \neq l$, that is equivalent to

$$
E\left[u\left(x+X_{t}^{N-3}\right)+E\left[u\left(x+X_{l}^{N-3}\right) \geq+E\left[u\left(x+X_{t}^{N-3}+X_{l}^{N-3}\right)\right]+u(x)\right.\right.
$$

which holds following $\operatorname{EST}(2009)$ theorem for all $u$ such that $(-1)^{(s+1)} u^{(s)}>0 \forall s=$ $1, \ldots, 2 k$ if $X_{1} \preceq_{S D_{k}} Y_{1}$ and $X_{2} \preceq_{S D_{k}} Y_{2}, k=1,2$. This inequality also holds following EST (2009) corollary for all $u$ such that $u^{(4)}<0$ in the particular case where risks are zero mean risks i.e. $X_{1} \preceq_{E k e r n_{2}} Y_{1}$ and $X_{2} \preceq_{E k e r n_{2}} Y_{2}$. 
In the expression $\sum_{i=1}^{N-(N-3)} E\left[u\left(x+X_{i}^{N-3}\right)\right]+E\left[u\left(x+X_{t}^{N-3}+X_{l}^{N-3}\right)\right]+(N-2) u(x)$, we have 2 risks $X_{i}^{N-2}\left(1\right.$ risk $X_{i}^{N-3}$ and 1 risk $\left.X_{t}^{N-3}+X_{l}^{N-3}\right)$. Thus it can be equivalently rewriten as $\sum_{i=1}^{2} E\left[u\left(x+X_{i}^{N-2}\right)\right]+(N-2) u(x)$ with $X_{i}^{N-2} \preceq_{S D_{k}} 0$ for all $k=1,2$ following the convolution stability property.

$$
\begin{aligned}
& \text { Step } N-1:\left(\left\{X_{i}\right\}_{N}^{2}\right) \succeq_{N-1}\left(\left\{X_{i}\right\}_{N}^{1}\right)(P(N-1)) \\
& (P(N-1)) \text { rewrites equivalently as } \\
& \sum_{i=1}^{2} E\left[u\left(x+X_{i}^{N-2}\right)\right]+(N-2) u(x) \geq E\left[u\left(x+X_{1}^{N-2}+X_{2}^{N-2}\right)\right]+(N-1) u(x) \text { which }
\end{aligned}
$$
holds following EST (2009) theorem for all $u$ such that $(-1)^{(s+1)} u^{(s)}>0 \forall s=1, \ldots, 2 k$ if $X_{1} \preceq_{S D_{k}} Y_{1}$ and $X_{2} \preceq_{S D_{k}} Y_{2}, k=1,2$. This inequality also holds following EST (2009) corollary for all $u$ such that $u^{(4)}<0$ in the particular case where risks are zero mean risks i.e. $X_{1} \preceq_{\text {Ekern }_{2}} Y_{1}$ and $X_{2} \preceq_{\text {Ekern }_{2}} Y_{2}$. 


\section{References}

Caballé, J. and Pomansky, A. (1996). 'Mixed risk aversion', Journal of Economic Theory, 71, 485-513.

Courbage, C., Loubergé, H. and Rey, B. (2018). 'On the properties of high-order non-monetary measures for risks', Geneva Risk and Insurance Review, 43, 77-94.

Denuit, M., Lefevre C., and Shaked, M. (1998). 'The s-convex orders among real random variables, with applications', Mathematical Inequalities and Application, 1(4), $585-613$.

Denuit, M., Lefevre, C., and Scarsini, M. (2001). 'On s-convexity and risk aversion'. Theory and Decision, 50(3), 239-248.

Ebert, S. (2013). 'Even (mixed) Risk Lovers are Prudent: Comment', American Economic Review, 103(4), 1536-1537.

Ebert, S., Nocetti, D.C. and Schlesinger, H. (2017). 'Greater mutual aggravation', Management Science, 64(6), 2473-2972.

Eeckhoudt, L. and Schlesinger, H. (2006). 'Putting risk in its proper place', American Economic Review, 96, 280-289.

Eeckhoudt, L., Schlesinger, H., and Tsetlin, E. (2009). 'Apportioning risks via stochastic dominance', Journal of Economic Theory, 113, 1-31.

Ekern, S. (1980). 'Increasing Nth degree risk', Economic Letters, 6, 329-333.

Friedman, M. and Savage, L.J. (1948). 'The utility analysis of choices involving risk', Journal of Political Economy, 56, 279-304.

Gollier, C. (1996). 'Decreasing absolute prudence: Characterization and applications to second-best risk sharing', European Economic Review, 40(9), 1799-1815.

Gollier, C. and Pratt, J.W. (1996). 'Risk vulnerability and the tempering effect of background risk', Econometrica, 64, 1109-1123.

Kimball, M.S. (1992). 'Precautionary motives for holding assets', in P. Newman, M. Milgate and J. Falwell (eds), The New Palgrave Dictionary of Money and Finance, London: Macmilan.

Menegatti, M. (2014). 'New results on the relationship among risk aversion, prudence and temperance', European Journal of Operational Research, 232, 613-617.

Nachman, D.C. (1982) 'Preservation of "More Risk Averse" under expectations', Journal of Economic Theory, 28, 361-368.

Rothschild, M., Stiglitz, J. (1970). 'Increasing risk: I. A definition', Journal of Economic Theory, 2, 225-243. 